\title{
Acute Fatty Liver of Pregnancy Showing Microbial Infection in the Liver
}

\author{
Mariko Kurosaki, Hitoshi Takagi, Yasuo Hosomura*, Jirou Takezawa, Masatomo Mori and Shigeharu Kudo**
}

\begin{abstract}
A 24-year-old, nulliparous woman in her 30th week of pregnancy was admitted due to threatened premature delivery. Ritodrin chloride relieved the premature contraction of the uterus but jaundice and drowsiness appeared 7 weeks later. Laboratory data revealed disseminated intravascular coagulation (DIC) with intrahepatic cholestasis, and ultrasound examination showed fatty liver. The patient was diagnosed with acute fatty liver of pregnancy (AFLP). Emergency delivery by Caesarean section was performed at 37 weeks of pregnancy and the liver function and DIC improved immediately. Liver biopsy 13 days after delivery showed nuclear swelling and cytoplasmic ballooning with mild fatty deposition. These findings were relatively compatible with acute AFLP. Higher magnification and electron microscopy revealed intracytoplasmic bacteria and fungus in the residual stage. The bacterial infection could be considered related to AFLP.

(Internal Medicine 39: 1064-1067, 2000)
\end{abstract}

Key words: AFLP, ultrastructure, bacteria, fungus

\section{Introduction}

Acute fatty liver of pregnancy (AFLP) sometimes causes fatal hepatic failure (1). Impaired mitochondrial oxidation of fatty acids has been thought to be the cause of AFLP (2), but the actual etiology has not been fully elucidated. Here we present a case of AFLP. The patient recovered after emergency delivery and liver biopsy showed fatty change accompanied by bacterial and fungal infection in the liver.

\section{Case Report}

A twenty-four-year-old, nulliparous woman in her 30th week of pregnancy complained of lower abdominal pain caused by uteral contractions. On April 19, 1993, she was diagnosed as being at risk for premature delivery. Ritodrin hydrochloride (15 mg per day) was administered to relieve the tension of the uterus. Six weeks later, she complained of nausea and vomiting and was admitted to the hospital on May 28.

The patient was of normal constitution-height, $159 \mathrm{~cm}$ and body weight, $62 \mathrm{~kg}$ and her consciousness was clear upon presentation. She showed a normal blood pressure of 124/60 $\mathrm{mmHg}$ and a regular pulse of 84 beats per minute. Her body temperature was 36.6 degrees Centigrade. No anemia, jaundice or lymphadenopathy were evident on admission. A chest examination showed no abnormal findings, and her liver was found to be soft and elastic under the right costal margin by palpation. No edema or abnormality was found in the lower extremities. Alkaline phosphatase was $293 \mathrm{IU} / \mathrm{l}$ (normal range 74-223), leucine aminopeptidase was $207 \mathrm{U} / \mathrm{l}(30-78)$ and total bilirubin $0.55 \mathrm{mg} / \mathrm{dl}$, alanine aminotransferase $13 \mathrm{IU} / \mathrm{l}$, and aspartate aminotransferase $11 \mathrm{IU} / l$. HBs antigen, anti-HBs antibody, anti-HBc-IgM antibody, anti-HCV antibody and antiHA-IgM were all negative. The patient experienced general fatigue, nausea, and vomiting at 37 weeks of pregnancy 1 week after the admission, and her liver dysfunction worsened to alanine transaminase of $127 \mathrm{IU} / l$, aspartate aminotransferase of $136 \mathrm{IU} / l$, alkaline phosphatase of $642 \mathrm{IU} / l$, leucine aminopeptidase of $342 \mathrm{IU} / l$, lactate dehydrogenase of $1,192 \mathrm{IU} / \mathrm{l}$ (220430), and total bilirubin of $10.8 \mathrm{mg} / \mathrm{dl} 1$ week later. Overt jaundice and drowsiness were also seen. The findings of elongated prothrombin time to 18.9 second, decreased fibrinogen of 113 $\mathrm{mg} / \mathrm{dl}$, anti-thrombin III of lower than $25 \mathrm{mg} / \mathrm{dl}$ (17-30), fibrinogen degradation product of more than $40 \mathrm{mg} / \mathrm{ml}$ and decreased platelet count of $9 \times 10^{4} / \mu 1$, increased levels of blood urea nitrogen of $20.4 \mathrm{mg} / \mathrm{dl}$ and creatinine of $4 \mathrm{mg} / \mathrm{dl}$ and uric acid of $11 \mathrm{mg} / \mathrm{d}$ l indicated a diagnosis of AFLP complicated with disseminated intravascular coagulation. A Caesarean section was performed on the day of diagnosis and the baby was born healthy. Total bilirubin increased to $17.6 \mathrm{mg} / \mathrm{dl}$ on June 21,1993 but it rapidly normalized together with the other laboratory data and the patient's general condition improved (Fig. 1). A liver biopsy performed 13 days after the delivery showed

From the First Department of Internal Medicine, Gunma University, School of Medicine, Maebashi, *the Department of Pathology, Sano-Kousei Hospital, Sano and **the First Department of Anatomy, Gunma University School of Medicine, Maebashi

Received for publication June 23, 1999; Accepted for publication July 3, 2000

Reprint requests should be addressed to Dr. Hitoshi Takagi, the First Department of Internal Medicine, Gunma University, School of Medicine, Maebashi, Gunma 371-8511 


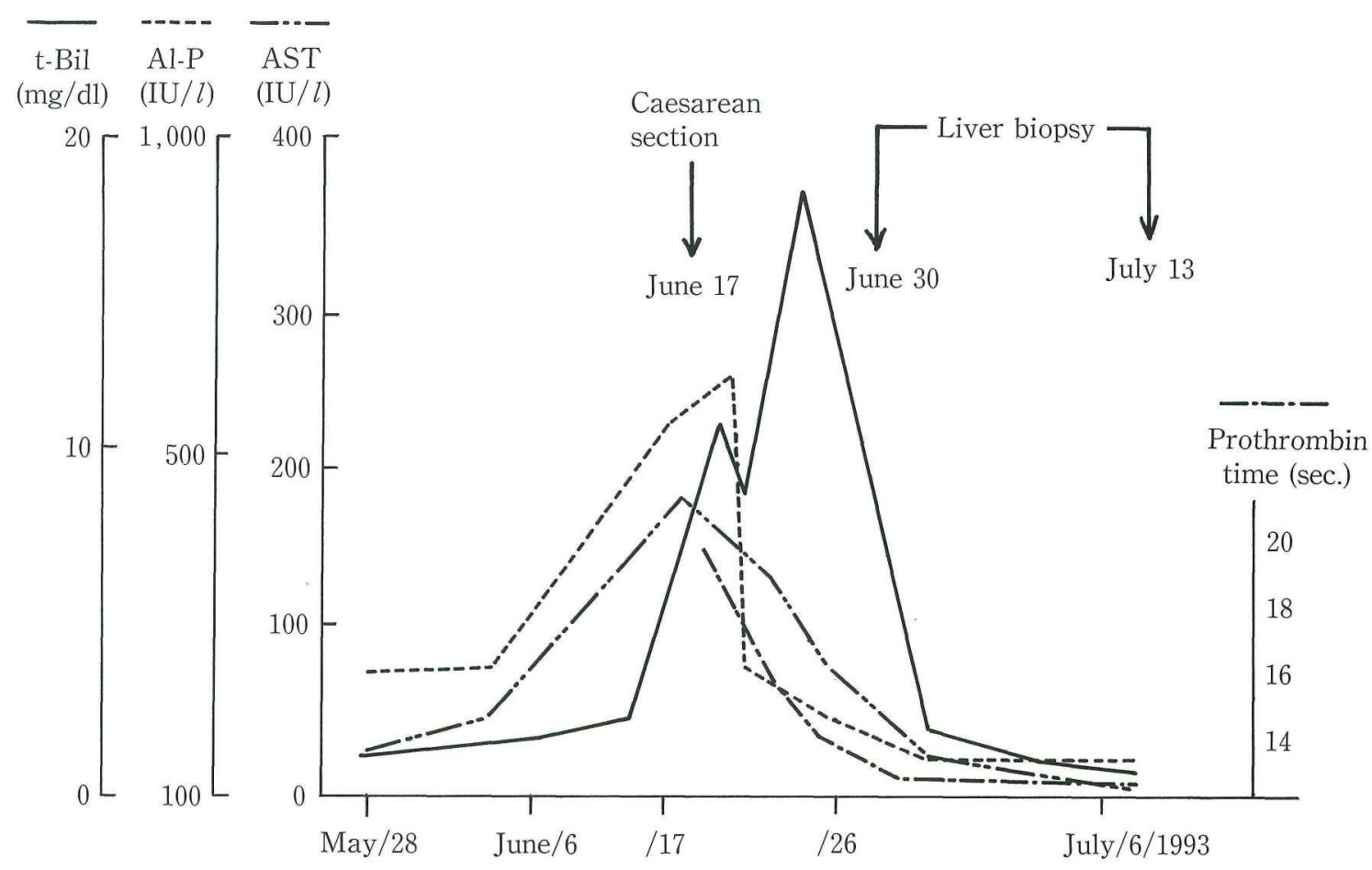

Figure 1. Clinical course of the patient. Al-P: alkaline phosphatase, AST: aspartate transaminase, t-Bil: total bilirubin.

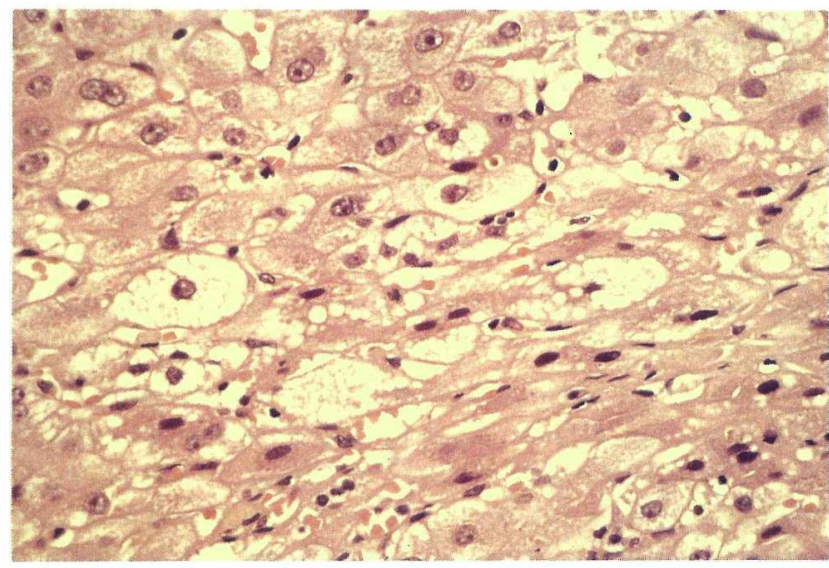

A

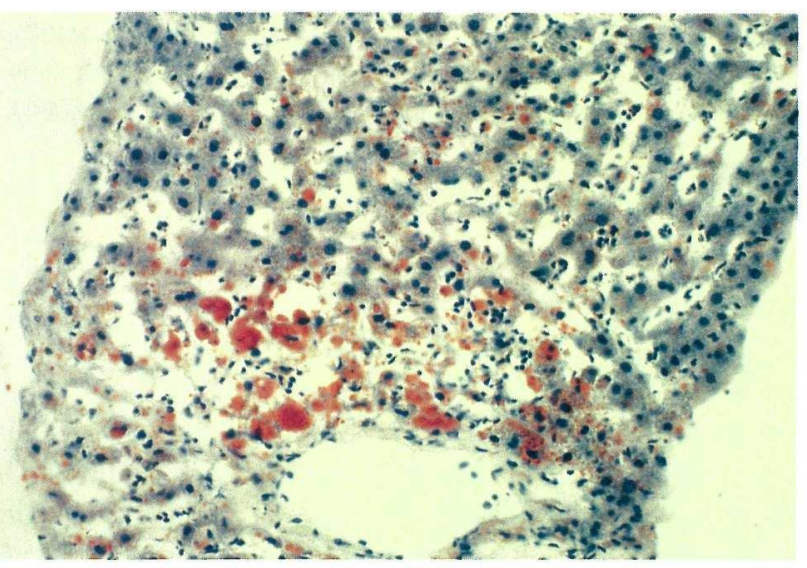

B

Figure 2. Liver biopsy performed 13 days after delivery. A) Nuclear swelling and cytoplasmic ballooning were observed (HE stain, $\times 200)$. B) Oil-red $O$ staining of the liver revealed small fatty infiltration, particularly around the central vein $(\times 100)$.

nuclear swelling and cytoplasmic ballooning (Fig. 2A). Oil redO staining demonstrated cytoplasmic fatty deposition predominant around the central vein (Fig. 2B). The second liver biopsy was performed 26 days after delivery and the residual stage of AFLP with bile plugs in bile ductule was noted (not shown). Highly magnified microscopic view and electron microscopic view showed fungal infection (Fig. 3A) and the presence of bacterial proliferation (Fig. 3B) although detailed bacterial classification was not available. The patient recovered from DIC and jaundice disappeared and she was discharged without any complications 1 month after the caesarian section. Bacterial and fungal infection could not have been the cause of AFLP but opportunistic infection seemed to be superimposed in the convalescent phase of AFLP. 


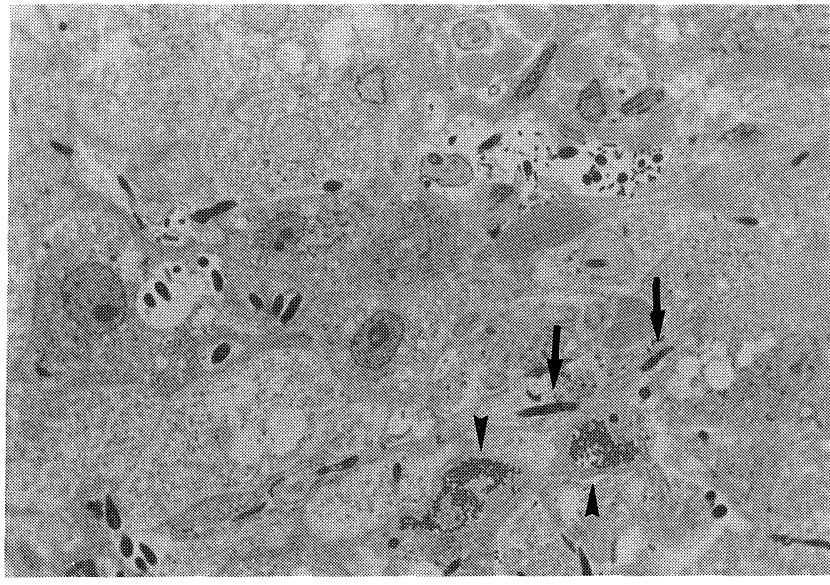

A

Figure 3. A) Higher magnification showed fungal infection (arrow) and bacterial proliferation (arrowhead) (HE stain, $\times \mathbf{4 0 0}$ ). B) Intracytoplasmic bacterial proliferation was shown on electron microscopy in hepatocytes 26 days after delivery $(\times 10,000)$.

\section{Discussion}

The present case of AFLP was relieved by emergency delivery by Caesarean section, and the baby was born healthy at 36 weeks of pregnancy. Although the cause of AFLP remains unknown, a deficiency of mitochondrial urea cycle enzyme activity has been demonstrated in several patients (3). In studies using animals, decreased mitochondrial $\beta$-oxidation of mediumchain fatty acids and decreased activity of the tricarboxylic acid cycle were accompanied by mitochondrial degeneration (2), and estrogen (4) was reported to cause mitochondrial dysfunction in pregnant rodents. In the present case, lipid deposition was scarcely visible by $\mathrm{HE}$ staining of hepatocytes at 13 days after delivery, but lipid-specific staining, e.g., oil-red O staining, was useful for detecting cytoplasmic lipid droplets, as previously reported (5). Electron microscopic examination is reported to be an important tool in the diagnosis of AFLP (5-7). Typical features of AFLP, such as osmophilic droplets of various size and megamitochondria containing crystalline inclusions (5) were not demonstrated in the present patient, but in-

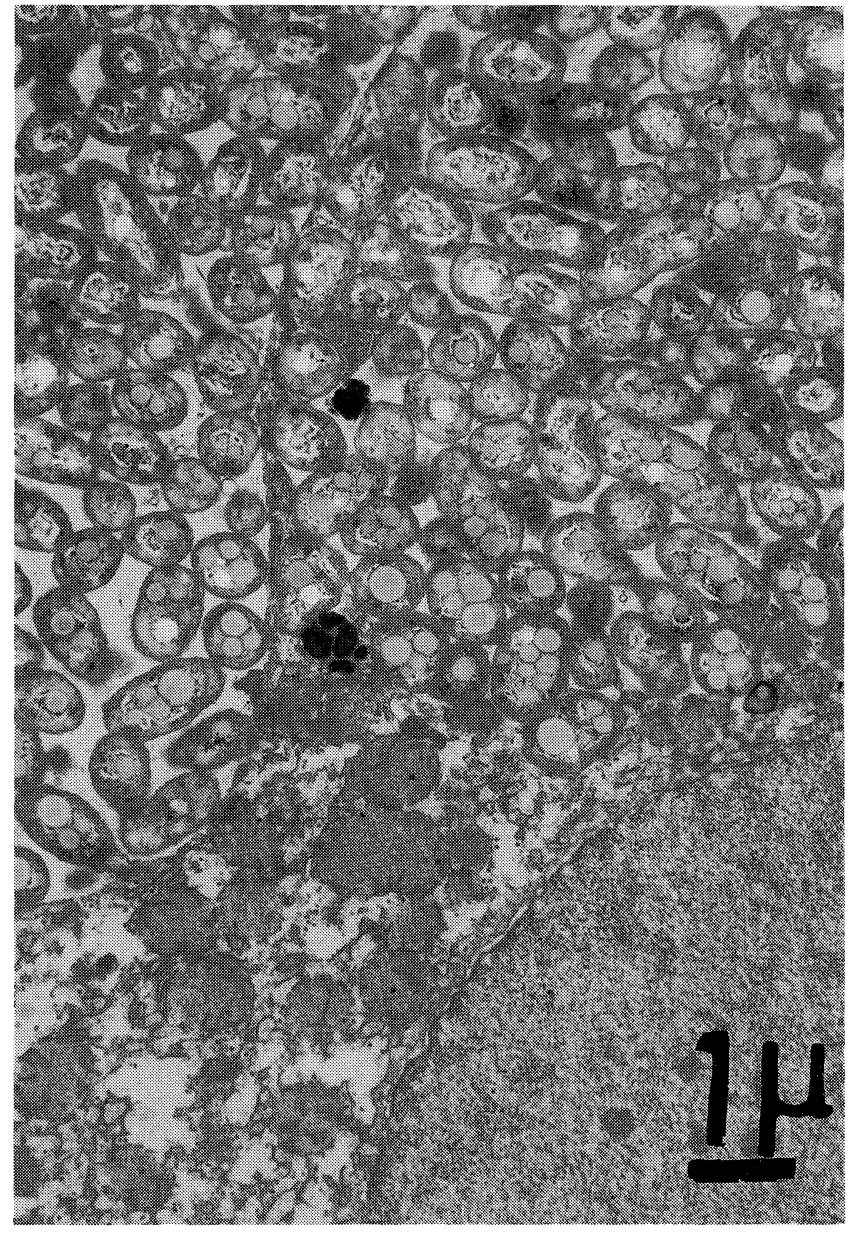

B

tracytoplasmic bacterial inclusion and fungal infection were identified. AFLP has never been reported to be caused by infection. Microscopic and ultrastructural findings of bacterial and fungal infection in the liver do not necessarily indicate the cause of AFLP but rather they are kinds of complications of AFLP. Although further studies will be needed to clarify the mechanism of AFLP, an infectious agent such as bacteria and fungus in the liver specimen may have some etiological contribution to AFLP.

As for the treatment of AFLP, Caesarian section was selected in this case, because the highest frequency of survival for both mother and baby is reported when delivery is made by Caesarian section or when delivery is induced (8). If symptoms of acute fatty liver of pregnancy are mild, careful fetal monitoring can be made in attempt to prolong gestation (9) but progression of AFLP is unpredictable. Liver failure induced by AFLP can be fully reversible if AFLP diagnosed early and if appropriate treatment such as induced delivery or Caesarian section was performed (10). Liver transplantation has been successful in the very rare cases in which supportive therapy following delivery does not bring about recovery (11). 


\section{Acute Fatty Liver of Pregnancy}

Acknowledgements: We thank Dr. T. Kamimura, The Second Saiseikai Niigata Hospital for interpreting the electron microscopic analysis.

\section{References}

1) Pereira SP, O'Donohue J, Wendon J, Williams R. Maternal and Perinatal outcome in severe pregnancy-related liver disease. Hepatology 26: 1258$1262,1997$.

2) Grimbert S, Fromenty B, Fisch C, et al. Decreased mitochondrial oxidation of fatty acids in pregnant mice: Possible relevance to development of acute fatty liver of pregnancy. Hepatology 17: 628-637, 1993.

3) Weber FL, Snodgrass PJ, Powell DE, Rao P, Huffman SL, Brady PG. Abnormalities of hepatic mitochondrial urea-cycle enzyme activities and hepatic ultrastructure in acute fatty liver of pregnancy. J Lab Clin Med 94: 27-41, 1979.

4) Grimbert S, Fisch C, Deschamps D, et al. Effects of female sex hormones on mitochondria: Possible role in acute fatty liver of pregnancy. Am $\mathrm{J}$ Physiol 268: G107-115, 1995.

5) Reyes H, Sandoval L, Wainstein A, et al. Acute fatty liver of pregnancy: a clinical study of 12 episodes in 11 patients. Gut 35: 101-106, 1994.

6) Rolfes DB, Ishak KG. Acute fatty liver of pregnancy: A clinicopathologic study of 35 cases. Hepatology 5: 1149-1 158, 1985.

7) Barton JR, Sibai BM, Mabie WC, Shanklin DR. Recurrent acute fatty liver of pregnancy. Am J Obstet Gynecol 163: 534-538, 1990.

8) Ebert EC, Sun EA, Wright SH, et al. Does early diagnosis and delivery in acute fatty liver of pregnancy lead to improvement in maternal and infant survival? Dig Dis Sci 29: 453-455, 1984.

9) Riely CA, Latham PS, Romero R, Duffy TP. Acute fatty liver of pregnancy. A reassessment based on observations in nine patients. Ann Intern Med 106: 703-706, 1987.

10) Castro MA, Fassett MJ, Reynolds TB, Shaw KJ, Goodwin TM. Reversible peripartum liver failure: a new perspective on the diagnosis, treatment, and cause of acute fatty liver of pregnancy, based on 28 consecutive cases. Am J Obstet Gynecol 181: 389-395, 1999.

11) Ockner SA, Brunt EM, Cohn SM, Krul ES, Hanto DW, Peters MG. Fulminant hepatic failure caused by acute fatty liver of pregnancy treated by orthotopic liver transplantation. Hepatology 11: 59-64, 1990. 